\title{
Patching and Tearing Single-Wall Carbon-Nanotube Ropes into Multiwall Carbon Nanotubes
}

\author{
María J. López, ${ }^{1, *}$ Angel Rubio, ${ }^{2}$ Julio A. Alonso, ${ }^{1}$ Serge Lefrant, ${ }^{3}$ Karine Méténier, ${ }^{4}$ and Sylvie Bonnamy ${ }^{4}$ \\ ${ }^{1}$ Departamento de Física Teórica, Universidad de Valladolid, E-47011 Valladolid, Spain \\ ${ }^{2}$ Departamento de Física de Materiales, Universidad del Pais Vasco, Centro Mixto CSIC-UPV/EHU \\ and Donostia International Physics Center (DIPC), Apartado 1072, 20018 San Sebastián, Spain \\ ${ }^{3}$ LPC/IMN, Université de Nantes, BP 32229, 44322 Nantes Cedex 3, France \\ ${ }^{4} C R M D, C N R S$-Université, $1 B$ rue de la Férollerie, 45071 Orléans Cedex 2, France
}

(Received 15 November 2001; published 27 November 2002)

\begin{abstract}
Bundles of single-wall carbon nanotubes (SWCNTs) coalesce forming multiwall carbon nanotubes (MWCNTs), containing from two to six nested tubes, under thermal treatment at high temperatures $\left[(2200-2400){ }^{\circ} \mathrm{C}\right]$. This structural transformation is confirmed by extensive molecular dynamics (MD) simulations. The simulations suggest a "patching-and-tearing" mechanism for the single-wall-tomultiwall transformation underlying the "concerted" coalescence of the tubes that begins with their polymerization. Tubes of different sizes and chiralities are considered.
\end{abstract}

DOI: $10.1103 /$ PhysRevLett.89.255501

PACS numbers: $61.46 .+\mathrm{w}, 65.80 .+\mathrm{n}$

Single-wall carbon nanotubes (SWCNTs) [1,2] and multiwall carbon nanotubes (MWCNTs) [3] are formed by $s p^{2}$ carbon atoms arranged in a graphitic honeycomb structure rolled up into seamless cylinders. Because of their metastable character, carbon nanotubes (CNTs) may transform into more stable structures under the appropriate "annealing" conditions. Investigations of the stability and annealing pathways of metastable structures are of primary importance in the context of technological applications. As some practical applications of CNTs are coming to reality, the need for a detailed and comprehensive characterization of the stability of CNTs is becoming imperative. Coalescence of SWCNTs annealed at high temperatures either in the presence of $\mathrm{H}_{2}$ [4] or under electron irradiation [5] has been observed. Coalescence of SWCNTs is a structural transformation leading to a more stable structure, i.e., a nanotube of larger diameter.

We have discovered a new thermally driven structural reorganization of SWCNTs leading to MWCNTs [6]. Extensive molecular dynamics simulations combined with the experimental results unveil the physical mechanism of this showy transformation based on the patching of SWCNTs and their subsequent tearing giving rise to MWCNTs. This novel mechanism, of general applicability, describes both the coalescence of two SWCNTs into a larger diameter SWCNT and the newest effect of the transformation of bundles of SWCNTs into MWCNTs. These results are supported by experiments on SWCNT ropes produced by the catalytic arc-discharge technique $[7,8]$. The samples were thermally treated (for about $15 \mathrm{~min})$ at temperatures between $1600-2800^{\circ} \mathrm{C}$ under argon flow and were characterized by high resolution transmission electron microscopy (HRTEM), x-ray diffraction, and Raman spectroscopy. These techniques confirm the disappearance of the bundles above $2200^{\circ} \mathrm{C}$ and the appearance instead of MWCNTs consisting of two to six nested tubes.
The samples treated at $1600^{\circ} \mathrm{C}$ show the same $\mathrm{x}$-ray pattern and the same Raman spectra as the untreated samples [6]. Only a better contrast in the HRTEM images was observed [see Fig. 1(a)]. After treatment at $1800^{\circ} \mathrm{C}$, the diameter of the individual tubes grew up from 1.4 to $2.0 \mathrm{~nm}$, which shows the beginning of coalescence. The breathing modes in the Raman spectrum (located in the range $140-190 \mathrm{~cm}^{-1}$ ) shifted a few percent towards lower frequencies. These modes are strongly dependent on tube diameter [6,9]. Moreover, the main peak in the small angle $\mathrm{x}$-ray scattering region shifted from $1.4 \mathrm{~nm}$ (position obtained with the untreated samples) to $1.72 \mathrm{~nm}$. This value gives directly the lattice parameter of the twodimensional arrangement of tubes in a bundle [10], showing an important expansion of the lattice. The samples treated at (2000-2200) ${ }^{\circ} \mathrm{C}$ show microtextural modifications; i.e., the nanotube diameters increase and the number of tubes per bundle decreases. Coalescence occurs readily in this temperature range. At $2000{ }^{\circ} \mathrm{C}$ most bundles consist of two to four tubes of $3 \mathrm{~nm}$ mean diameter [Fig. 1(b)] and, accordingly, the low frequency modes in the Raman spectrum shifted further towards lower

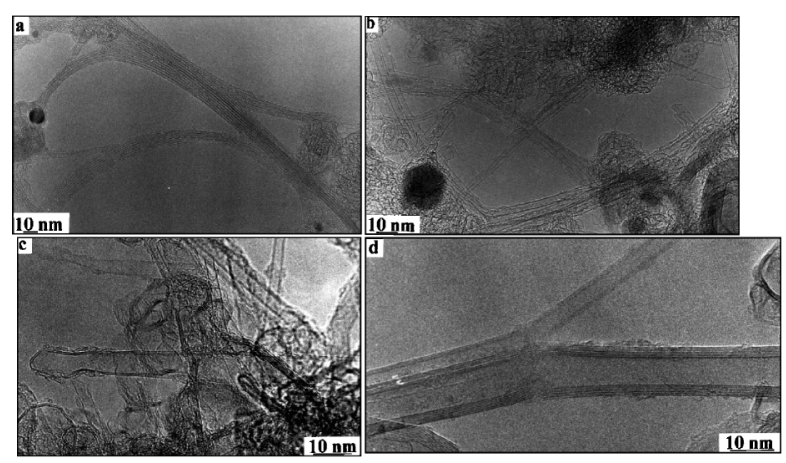

FIG. 1. HRTEM image of the sample of SWCNTs after heat treatment at (a) $1600{ }^{\circ} \mathrm{C}$, (b) $2000{ }^{\circ} \mathrm{C}$, (c) $2400{ }^{\circ} \mathrm{C}$, and (d) $2800{ }^{\circ} \mathrm{C}$ (see text for description). 
frequencies forming a band distribution. At $2200^{\circ} \mathrm{C}$, no more bundles are observed, only short isolated SWCNTs of about $4 \mathrm{~nm}$ in diameter associated to the first MWCNTs are present, and, consequently, the low frequency features of the Raman spectrum almost disappeared. Incidentally, the catalyst vaporizes completely at this temperature. At $2400{ }^{\circ} \mathrm{C}$, very few isolated SWCNTs remain and instead many MWCNTs consisting of two to six nested tubes appear [Fig. 1(c)]. The disappearance of the SWCNTs is confirmed by the disappearance of the small angle peaks in the $\mathrm{x}$-ray diffraction pattern and by the disappearance of the low frequency Raman modes, fingerprint of the SWCNTs. Above $2400{ }^{\circ} \mathrm{C}$, only MWCNTs are observed with an external diameter of 10-12 nm [Fig. 1(d)]. The high frequency region of the Raman spectrum also supports the SW-to-MW transformation. The spectrum shows the disappearance at about $2200{ }^{\circ} \mathrm{C}$ of the typical double peak around $1580 \mathrm{~cm}^{-1}$ due to the $G$ modes of the SWCNTs and the appearance of a broad line at $1577 \mathrm{~cm}^{-1}$ with a small wing at $1600 \mathrm{~cm}^{-1}$ typical of MWCNTs. Moreover, the appearance of the $D$ band at $1345 \mathrm{~cm}^{-1}$ implies the presence of tubes with defects.

We have investigated the coalescence and, more importantly, the stability and temperature induced SW-toMW transformation. A many-body interatomic potential is used to mimic the carbon-carbon interactions. This potential includes coordination and angular dependence and is well suited for describing both the intratube covalent bonds [11] and the van der Waals interactions between adjacent tubes [12]. It has proven reliable in the description of a wide variety of carbon systems and their properties, including CNTs [13] and bond breaking and bond rearrangement in tubes [5]. The extent of the simulations presented in this letter, with about 6000 inequivalent carbon atoms and time scales of the order of $1000 \mathrm{ps}$, makes the use of more accurate quantum techniques (ab initio or semiempirical) impractical if not completely unfeasible. Moreover, the mechanical and thermal characteristics of the tubes involved in the description of the SW-to-MW transformation are not very sensitive to the fine details of the electronic structure of the tubes since they are common to different types of tubes. Therefore, the general features of the simulation should remain valid even if first-principles simulation techniques were used. The "patching-and-tearing" mechanism underlying the coalescence of the tubes and the SW-to-MW structural reorganization, through a concerted coalescence of the tubes, is analyzed in detail as well as the conditions for the successful coalescence of two tubes. It has been stated that coalescence of tubes of different chiralities is unlikely since large rearrangements of atoms are required for matching the two tubes. In contrast to those arguments, we find no restrictions for the coalescence of tubes with different chiralities, provided that sufficiently large simulation cells are considered (see below).
It has been found that vacancies are the leading defects promoting coalescence [5] through an initial stage of intertube polymerization. We then assume (this assumption will be lately confirmed by the simulations) that vacancies or defects (structural, impurities, chemically induced, ... ) also play a dominant role in the SW-to-MW transformation of tubes. The first step of the transformation consists in the generation of defects in the tubes, in particular, vacancies. The thermal treatment of the bundles at relatively high temperatures (above $1600^{\circ} \mathrm{C}$ ) will produce the required vacancies in the tubes. At these temperatures, the mobility of the defects throughout the tubes is high. The vacancies will then migrate until they get trapped in the intertube regions by saturation of the dangling bonds they leave free with the dangling bonds left free by another vacancy in the adjacent tube, giving rise to the polymerization of the tubes. The time scale for this first step (about minutes at the experimental temperatures) is not tractable in conventional MD simulations. Once the vacancies have been generated and are trapped in the intertube regions, the structural reorganization of the tubes occurs within a time scale of a few hundred picoseconds. This is a suitable time for being simulated in the computer. For this reason we concentrate our study on this second step of the SW-to-MW process.

We first simulate a bundle of seven $(10,10)$ tubes arranged in a triangular lattice (with lattice parameter of $17 \AA$ ) with a central tube and six surrounding tubes (see Fig. 2). The simulation cell contains 20 unit cells in the axial direction of the tubes (5600 $\mathrm{C}$ atoms) and is
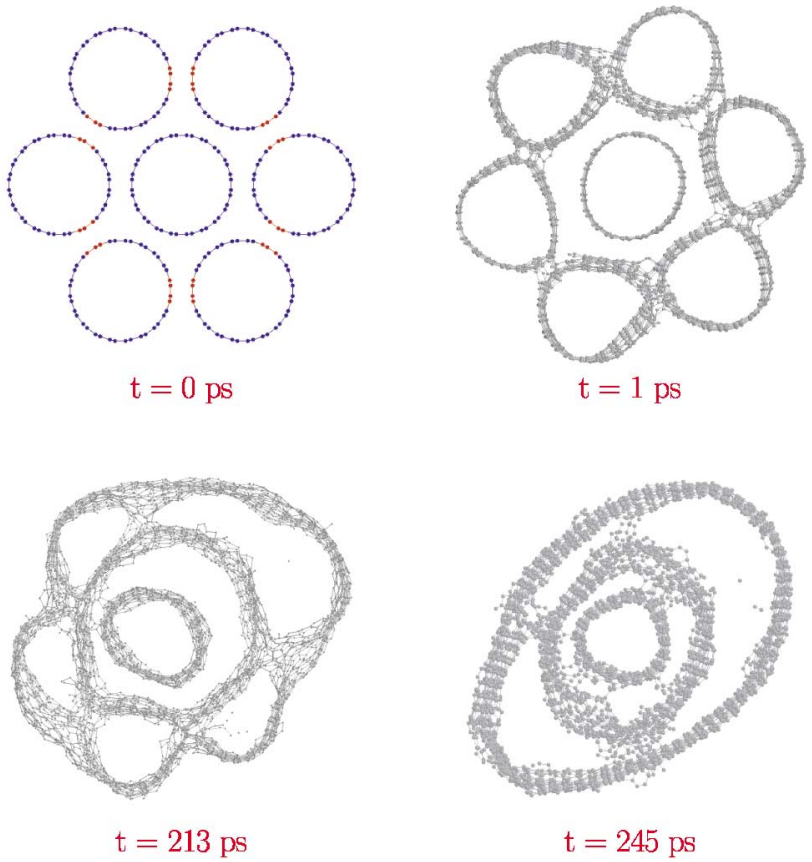

FIG. 2 (color online). Snapshots (top view) taken from the simulations showing the sequence of the SW-to-MW transformation of a bundle consisting of seven $(10,10)$ SWCNTs (see text for details). 
repeated in this direction using periodic boundary conditions. The simulation begins with the random generation of a number (4\%) of vacancies in the six outer tubes (no defects are generated in the central tube; see below for a justification). We restrict the removal of $\mathrm{C}$ atoms to the intertube regions (Fig. 2, $t=0 \mathrm{ps)} \mathrm{since,} \mathrm{given} \mathrm{the} \mathrm{long}$ time scale of the diffusion of vacancies along the tubes, only those defects in the vicinity of neighboring tubes play an active role in the structural transformation. Polymerization between neighboring tubes occurs in less than $1 \mathrm{ps}$ after the generation of vacancies (Fig. 2, $t=1 \mathrm{ps}$ ) driven by the saturation of the dangling bonds left free by the vacancies. The bundle is then heated up from room temperature up to a sufficiently high temperature [14] to accelerate the coalescence process up to a time scale shorter than $1 \mathrm{~ns}$. The intertube links evolve into "patches," i.e, portions of an enveloping surface common to the two tubes. The patches grow and merge in the axial direction of the tubes until they cover the whole tube length. The coalescence of two neighboring tubes finishes with the tearing apart of the remaining intratube bonds in the intertube region (Fig. 2, $t=213$ ps). Finally, the concerted patching and tearing of all the tubes around the bundle gives rise to a MWCNT consisting of three nested tubes (Fig. 2, $t=245 \mathrm{ps}$ ). It is fair to notice that, even if the number of defects in the experiment would be smaller than in the simulations, this would imply only a change in the time scale for coalescence not in the coalescence mechanism. The central tube may also have a number of defects and establish links with the surrounding tubes. Then, besides the three nested structures shown here (Fig. 2), two nested structures may also occur. On the other hand, the excellent agreement between simulations and experiments gives us confidence that the somewhat higher temperatures used in the simulations to bring the rate of the coalescence process to a time scale attainable in the simulations do not modify its mechanism.

The MW tubes just formed have many defects mainly along the patching lines between the original tubes. Annealing of those defects will occur in a time scale not reachable in simulations although it will certainly occur in the experiment. The heat produced during the coalescence leads to the evaporation of a number of carbon atoms and little chains from the tube walls. Some of them are lost into the vacuum and some diffuse, along the tube axis, in the interior of the tubes until, eventually, sticking to one defect of the tube. Those diffusing atoms and clusters constitute a useful restoring mechanism of the tube surface upon coalescence.

A similar patching-and-tearing mechanism operating in a concerted way in a bundle of four $(10,10)$ tubes gives rise to a MWCNT consisting of two nested tubes. In contrast, a bundle of three $(10,10)$ tubes coalesces into a single-wall tube. The reason could be that the three SW tubes do not supply enough $\mathrm{C}$ atoms to reconstruct an internal tube. However, a bundle of three $(20,20)$ tubes [where the number of $\mathrm{C}$ atoms is sufficient to reconstruct an internal $(9,9)$ tube] also coalesces into a larger singlewall tube. This seems to indicate that a minimum of four tubes is needed to observe the transformation into MWCNTs. Moreover, our simulations of the coalescence of two $(10,10)$ tubes agree with the tight-binding simulation results of Terrones et al. [5].

To further validate the patching-and-tearing mechanism, we have simulated the coalescence of two tubes using a much longer simulation cell consisting of 60 unit cells in the axial direction of the tubes (see Fig. 3). The size of the simulation cell is an important parameter because, since no diffusion of vacancies is expected within the simulation time, it is the initial distribution of vacancies which largely determines the subsequent evolution of the system. Short simulation cells result in a uniform distribution of vacancies due to the periodic boundary conditions. Therefore, longer cells should be considered to have a truly random nonuniform distribution of vacancies mimicking the experimental situation. To initiate the simulation, a number (120) of $\mathrm{C}$ atoms are removed at random from the intertube region. Similarly to the simulations with the shorter cell, the tubes polymerize immediately after the generation of vacancies (Fig. 3, $t=2$ ps). However, the intertube links do not appear uniformly distributed along the tube length. Upon heating [14], patches enveloping the two tubes develop in some portions of the tubes and some of them tear giving rise to partial coalescence of the tubes, whereas other portions along the tubes remain unlinked or just polymerized (Fig. 3, $t=125 \mathrm{ps}$ ). The coalesced parts of the tubes and the patches grow in the axial direction as the time evolves and new patches also form
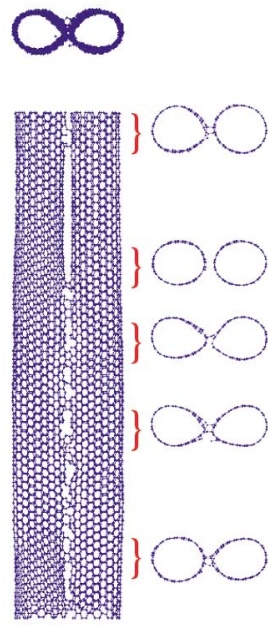

$\mathrm{t}=2 \mathrm{ps}$
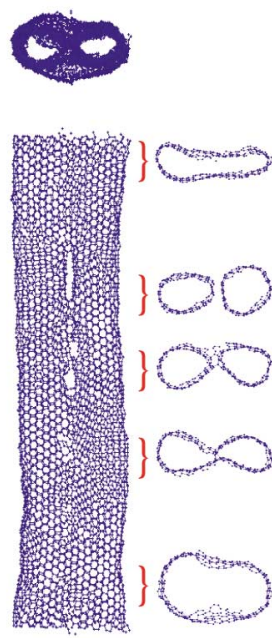

$\mathrm{t}=125 \mathrm{ps}$
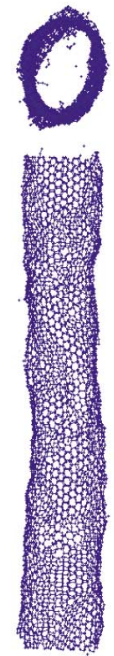

$\mathrm{t}=165 \mathrm{ps}$
FIG. 3 (color online). Snapshots (side and top views) taken from the simulations showing the sequence of the coalescence of two $(10,10)$ SWCNTs. Top views of some portions of the tube length (embraced by brackets) are also shown next to the corresponding brackets (see text for details). 
and merge with other patches. Eventually, the two tubes coalesce in their whole length (Fig. $3, t=165 \mathrm{ps)}$. The patching-and-tearing mechanism described here explains in great detail the process of coalescence of two tubes. This mechanism complements and gives support for the intuitive description of coalescence through a zipping process [4,5].

The possible coalescence of two tubes with different chiralities has been investigated for tubes with similar radii, e.g., a $(10,10)$ tube (radius $R=6.98 \AA$ ) and a $(12,8)$ tube $(R=7.03 \AA)$. Since the unit cells of tubes with different chiralities are not commensurate in general, we have chosen a sufficiently large simulation cell (171.96 $\AA$ in length) which approximately contains an integer number of unit cells of both tubes (68 and 9 unit cells, respectively). Coalescence of the two tubes takes place following the patching-and-tearing mechanism in a similar manner as in the case of two nonchiral tubes. The most stringent case of two nonchiral tubes, one of the armchair type, the $(10,10)$ tube, and the other one of the zigzag type, the $(17,0)$ tube, has also been checked. The two tubes coalesce following the same patterns as before. From our simulations we conclude, in contrast with the arguments of Terrones et al. [5] that there are no constraints for coalescence of tubes with different chiralities. One should notice, however, that large simulation cells are required to observe coalescence of tubes of different chiralities due to the mismatch of their respective honeycomb structures. Moreover, the resulting coalesced tube has a higher density of defects (which are not annealed within the limited time of our simulations) than in the case of tubes with the same chirality.

It is interesting to stress that the SW-to-MW transformation proceeds in two steps which involve very different time scales. The first step consists in the generation of defects (vacancies) and their migration towards the intertube regions where they get trapped by saturation of the associated dangling bonds giving rise to intertube polymerization. This process may take several minutes at the experimental temperatures. The second step, i.e., the structural reorganization of the tubes, occurs very fast (in a few hundred ps). In the MD simulations, upon the generation of vacancies, intertube links appear readily, producing the polymerization of tubes in the bundle. Then, the adjacent tubes patch together by generating a common enveloping wall and, finally, the remaining intratube bonds in the intertube regions tear apart, giving rise to the multiwall tube. Therefore, the SW-to-MW transformation takes place through a concerted patchingand-tearing mechanism. This mechanism gives support to the more intuitive zipping mechanism which was used to explain the coalescence of tubes observed under $\mathrm{H}_{2}$ treatment or under electron irradiation. The observed thickening of the MWCNT heat treated at $2800^{\circ} \mathrm{C}$ [see Fig. 1(d)] may occur by the growth and adjunction of graphitic layers on the surface of the MWCNTs formed in the earlier stage.
Bundles of SWCNTs are stable, under thermal treatment, up to $1600{ }^{\circ} \mathrm{C}$. Above $T=2200{ }^{\circ} \mathrm{C}$, they become unstable and transform into MWCNTs. This temperature is in fair agreement with the graphitization temperature of carbon materials [15]. However, at those temperatures vaporization of the carbon atoms is not a relevant factor, which suggests that the formation mechanism of MWCNTs from SWCNT bundles is not the same as that of natively grown MWCNTs. Further studies are needed to improve our understanding of the structural stability, their possible morphological changes, and the correlation, if any, with the various growth mechanisms [16] of CNTs.

This work was supported by DGES (Grant No. PB980345), European Community (RTN-COMELCAN HPRN-CT-2000-00128 and SATUNET IST-200026361), and Junta de Castilla y León (CO 01/102). We acknowledge the computational facilities provided by CESCA and CEPBA.

*Electronic address: maria@rhodas.fam.cie.uva.es

[1] S. Iijima and T. Ichihashi, Nature (London) 363, 603 (1993).

[2] D. S. Bethune et al., Nature (London) 363, 605 (1993).

[3] S. Iijima, Nature (London) 354, 56 (1991).

[4] P. Nikolaev et al., Chem. Phys. Lett. 266, 422 (1997).

[5] M. Terrones et al., Science 288, 1226 (2000).

[6] M. Lamy de la Chapelle et al., in Molecular Nanostructures, edited by H. Kuzmany, J. Fink, M. Mehring, and S. Roth (World Scientific, Singapore, 1998), p. 387.

[7] C. Journet et al., Nature (London) 388, 756 (1997).

[8] HRTEM characterization of the sample shows that it is made of bundles of SWCNTs with bundle diameters ranging from 5 to $20 \mathrm{~nm}$. The individual tubes of about $1.4 \mathrm{~nm}$ in diameter are arranged in a triangular lattice.

[9] P. C. Eklund, Carbon 33, 959 (1995).

[10] A. Thess et al., Science 273, 483 (1996).

[11] J. Tersoff, Phys. Rev. B 37, 6991 (1988); Phys. Rev. Lett. 61, 2879 (1988).

[12] K. Nordlund, J. Keinonen, and T. Mattila, Phys. Rev. Lett. 77, 699 (1996).

[13] See, e.g., M. B. Nardelli et al., Phys. Rev. Lett. 80, 313 (1998); M. J. López et al., ibid. 86, 3056 (2001).

[14] The bundles are heated up from room temperature up to about $3200^{\circ} \mathrm{C}$ at an average rate of $150{ }^{\circ} \mathrm{C}$ per $10 \mathrm{ps}$ and then the temperature is kept approximately constant. The simulation temperatures reported here refer to the kinetic temperatures scaled down by a factor of 0.7 to reproduce the experimental melting temperature of graphite $\left(T_{m}=\right.$ $4300 \mathrm{~K})$.

[15] A. Oberlin, in Chemistry and Physics of Carbon, edited by P. A. Thrower (Dekker, New York, 1989), Vol. 22, p. 1; A. Oberlin and S. Bonnamy, in The World of Carbon: Graphite and Precursors, edited by P. Delhaes (Gordon and Breach, New York, 2001), Vol. 1, p. 199.

[16] J. Gavillet et al., Phys. Rev. Lett. 87, 275504 (2001). 